Article

\title{
Nanoparticle Determination in Water by LED-Excited Surface Plasmon Resonance Imaging
}

\author{
Lukas Wunderlich ${ }^{1}$, Peter Hausler ${ }^{2}$, Susanne Märkl ${ }^{1}$, Rudolf Bierl ${ }^{2}$ and Thomas Hirsch ${ }^{1, * \text { (D) }}$ \\ 1 Institute of Analytical Chemistry, Chemo- and Biosensors, University of Regensburg, Universitätsstraße 31, \\ 93040 Regensburg, Germany; lukas.wunderlich@ur.de (L.W.); susanne.maerkl@ur.de (S.M.) \\ 2 Sensorik-ApplikationsZentrum, Ostbayerische Technische Hochschule Regensburg, Franz-Mayer-Str. 1, \\ 93053 Regensburg, Germany; peter.hausler@oth-regensburg.de (P.H.); rudolf.bierl@oth-regensburg.de (R.B.) \\ * Correspondence: thomas.hirsch@ur.de
}

check for updates

Citation: Wunderlich, L.; Hausler, P.; Märkl, S.; Bierl, R.; Hirsch, T.

Nanoparticle Determination in Water by LED-Excited Surface Plasmon Resonance Imaging. Chemosensors 2021, 9, 175. https://doi.org/ $10.3390 /$ chemosensors 9070175

Academic Editor: Raffaele Velotta

Received: 18 June 2021

Accepted: 7 July 2021

Published: 11 July 2021

Publisher's Note: MDPI stays neutral with regard to jurisdictional claims in published maps and institutional affiliations.

Copyright: (c) 2021 by the authors. Licensee MDPI, Basel, Switzerland. This article is an open access article distributed under the terms and conditions of the Creative Commons Attribution (CC BY) license (https:/ / creativecommons.org/licenses/by/ $4.0 /)$.

\begin{abstract}
The increasing popularity of nanoparticles in many applications has led to the fact that these persistent materials pollute our environment and threaten our health. An online sensor system for monitoring the presence of nanoparticles in fresh water would be highly desired. We propose a label-free sensor based on SPR imaging. The sensitivity was enhanced by a factor of about 100 by improving the detector by using a high-resolution camera. This revealed that the light source also needed to be improved by using LED excitation instead of a laser light source. As a receptor, different self-assembled monolayers have been screened. It can be seen that the nanoparticle receptor interaction is of a complex nature. The best system when taking sensitivity as well as reversibility into account is given by a dodecanethiol monolayer on the gold sensor surface. Lanthanide-doped nanoparticles, $29 \mathrm{~nm}$ in diameter and with a similar refractive index to the most common silica nanoparticles were detected in water down to $1.5 \mu \mathrm{g} \mathrm{mL}^{-1}$. The sensor can be fully regenerated within one hour without the need for any washing buffer. This sensing concept is expected to be easily adapted for the detection of nanoparticles of different size, shape, and composition, and upon miniaturization, suitable for long-term applications to monitor the quality of water.
\end{abstract}

Keywords: surface plasmon resonance; imaging; nanoparticle; sensor

\section{Introduction}

Nanoparticles are becoming more and more of an everyday material, as they find applications as catalysts in petroleum refining, as surface coatings in displays and optical glasses, in paints, as smart fabrics with antimicrobial properties in textiles, as drug delivery systems in biomedicine, or in cosmetics. The most prominent nanoparticles are made from gold, silver, iron, lanthanides, $\mathrm{TiO}_{2}$, and silica. The European Community reports a production of about 1.5 million tons per year of silica particles [1], and a mean concentration of $5.34 \mu \mathrm{g} \mathrm{L}^{-1}$ is predicted to be found in fresh surface water [2]. The potential risk of nanoparticle pollution has been known for more than a decade. The sheer number of different nanoparticles [3], in addition to their prevalent use, led to the assumption of possible health-related problems [4,5]. In addition to that, no or little information is available about the health risks of nanoparticles after their release to nature and potential subsequent degradation [3,6]. A major problem, besides their complete removal, is the challenging detection of these small particles over a widespread area in complex media to identify the nanoparticle paths over their lifespan [5,6]. As a result of the increasing concentration and subsequent health risks of nanoparticle pollution, different techniques have been investigated to enable the widespread monitoring of them and provide insights into their distribution and concentration. Detection methods such as inductively coupled plasma combined with mass spectrometry (ICP-MS) [7], surface enhanced Raman spectroscopy $[7,8]$ or electrochemical techniques [7,9] have been tailored to be suitable for nanoparticle detection, all with different advantages and disadvantages. ICP-MS shows a 
high sensitivity but needs elaborate equipment and a high amount of labor. Measurements based on surface enhanced Raman spectroscopy and electrochemistry can enable an online and miniaturized setup, but certainly still require sample pretreatment and are confined to a selection of particles. This clearly demonstrates the need to develop sensors suitable for the online monitoring of nanoparticles in the environment.

Surface plasmon resonance (SPR) might be a sufficient tool for this purpose. The excitation of surface plasmons on a gold surface deposited on a prism by a monochromatic light source is highly sensitive to changes in its surrounding environment [10-13]. Nanoparticles, which are large sized analytes, compared to other pollutants such as chemicals or pharmaceutics, generate a larger signal change in SPR, which allows us to detect them at relative low levels [14-17] or use them as labels for signal enhancement [18,19]. The advantage of using an SPR imaging (SPRi) setup compared to a single channel SPR is the ability to easily functionalize the sensor surface by a variation of receptors, enabling the determination of multiple analytes at once [20,21]. Reflectivity-based SPRi setups have been reported to resolve about $10^{-5}$ refractive index units (RIU) [22]. There were many tries to overcome this limit by developing advanced SPRi technologies such as spectral SPRi or phase contrast SPRi [23]. However, these technologies all had disadvantages, such as a more complex setup, which impedes a widespread sensor implementation. The sensitivity of reflectivity-based SPRi can be enhanced by the implementation of a more sensitive and temperature-stabilized camera of a high-resolution (16-bit and better) [24]. While having a more sensitive detector, the properties of the light source became more important. Point-based SPR predominantly uses lasers as a light source. This is because most semiconductor lasers are polarized and emit at a wavelength with a very narrow full width half maximum (FWHM). In addition to such an advantage, laser light sources in SPR suffer from a spatial coherence that generates diffraction patterns from every object within the light beam. Hence, every dust particle or every structure at the sensing surface will generate diffraction patterns. Moreover, the quality of the retrieved SPR image in prism-coupled sensors is affected by the creation of speckles, which arise from the roughness at the interface of the glass to the metal layer, when it is in the range of the excitation wavelength or higher. Furthermore, semiconductor lasers lack a temperature-dependent wavelength shift and they can even demonstrate mode hopping. To overcome these problems and enable the full potential of a high-resolution camera, the laser was exchanged with a high-power LED, which enables a homogenized intensity over the whole sensor surface and low noise [25]. To enable the required selectivity as well, the SPR prism was functionalized with differently capped alkanethiols, providing different surface charges as receptor elements. Their low selectivity is ideal for an application in a cross-reactivity sensor system in the future, capable of detecting the huge variety of different nanoparticles. Lanthanide-doped yttrium fluoride particles have been chosen as model particles in this work. First, they belong to non-metallic particles, and therefore cannot be easily detected by electrochemical stripping methods [5], which makes them ideal candidates to demonstrate the power of SPR-based sensing. Second, those particles can be tailored very reproducibly regarding their size, with extremely narrow size distribution. Their refractive index of $\sim 1.475$ [26] is nearly identical to the widely applied silica nanoparticle $\left(\mathrm{n}_{\mathrm{D}}=1.475,[27]\right)$, which, in contrast to the yttrium fluoride particles, tend to form aggregates, and therefore are not as ideal as model analyte particles. With this study, we demonstrate the feasibility of the reversible detection of the presence of nanoparticles in water by using an improved SPRi setup.

\section{Materials and Methods}

6-amino-1-hexanethiol hydrochloride, 16-mercaptohexadecanoic acid $(90 \%, w / w)$, 1-dodecanthiol $(98 \%, w / w)$ and ethanol (absolute, $>99.8 \%, w / w)$ were purchased from Sigma-Aldrich (Taufkirchen, Germany). $\mathrm{NaCl}$ salt $(>99.5 \%, w / w)$, glucose (p.a.) and ethanol (p.a., $\geq 99.8 \%, w / w$ ) were obtained from Carl Roth (Karlsruhe, Germany). The poly(dimethylsiloxane) (PDMS) stamp was fabricated using Sylgard 184 silicone elastomer 
base and curing agent by Dow Corning (Wiesbaden, Germany) in a ratio of 10:1 $(v / v)$. All reagents were used without further purification. Double distilled water was used in all experiments.

Oleate-coated core-shell nanoparticles $\mathrm{NaYF}_{4}(\mathrm{Yb}, \mathrm{Er}) @ \mathrm{NaYF}_{4}$ were prepared by means of a protocol published recently [28] in a two-step approach. Firstly, core particles were synthesized from the rare earth trichlorides in high boiling solvents with $78 \% \mathrm{YCl}_{3}, 20 \% \mathrm{YbCl}_{3}$ and $2 \% \mathrm{ErCl}_{3}$. Cubic $\mathrm{NaYF}_{4}$ particles were used to grow shells around the core particles through a stepwise injection of the cubic particles to a boiling dispersion of the hexagonal particles. The oleate coating of the core-shell nanoparticles $\mathrm{NaYF}_{4}(\mathrm{Yb}, \mathrm{Er}) @ \mathrm{NaYF}_{4}$ was exchanged for $\mathrm{BF}_{4}{ }^{-}$[29], and the $\mathrm{BF}_{4}{ }^{-}$-coated nanoparticles were dispersed in $\mathrm{H}_{2} \mathrm{O}$. The concentration was determined by inductively coupled plasma optical emission spectrometry (ICP-OES) with a Spectro Flame-EOP (Kleve, Germany).

A commercially available BioSuplar SPR 521i instrument (Mivitec GmbH, Sinzing, Germany) was used, equipped with an F1-65 glass prism installed on a swivel carriage. F1 gold-coated glass slides $(20 \mathrm{~mm} \times 20 \mathrm{~mm}$ ) were obtained from Mivitec $\mathrm{GmbH}$ (Sinzing, Germany).

The homemade SPRi setup was built with a V-shaped light path with fixed arms and a movable middle edge on a linear stage, to adjust the measurement angle (step size $0.001^{\circ}$ ). An OSLON SQUARE $660 \mathrm{~nm}$ LED was purchased from Osram GmbH (Berlin, Germany). The LED was driven by a 2636B SourceMeter by Keithley (Cologne, Germany) at a current of $10 \mathrm{~mA}$. Further implemented optical components were an $\mathrm{f} 40$ aspheric plastic lens and a 1:9000 ratio polarizer, from Edmund Optics (York, UK), and a $2 \mathrm{~nm}$ bandwidth filter. The laser reference measurements were performed using a $660 \mathrm{~nm}, 75 \mathrm{~mW}$ miniLas from RGB Lasersystems (Kelheim, Germany), which was run at a power of $10 \mathrm{~mW}$. The image is acquired by a 16-bit sCMOS Camera Edge 4.2 from PCO AG (Kelheim, Germany).

Schott F2 glass prisms were installed in this setup. Before usage, the prism surface was polished to grade $\mathrm{P} 4$, and an approximately $1 \mathrm{~nm}$ chromium adhesive layer and an approximately $50 \mathrm{~nm}$ gold layer were deposited on top by electron beam deposition.

The microfluidics consisted of a micropump, purchased from Bartels Mikrotechnik $\mathrm{GmbH}$ (Dortmund, Germany) and a custom-made microfluidic chip, made of stainless steel.

The refractive index of the solutions was determined with a DR6300-T refractometer from Krüss (Hamburg, Germany). Dynamic light scattering and zeta potential measurements have been performed with a Nano ZS from Malvern (Worcestershire, UK) at $20{ }^{\circ} \mathrm{C}$. Transmission electron microscopy (TEM) was used to estimate the nanoparticle size. Samples on carbon-coated copper grids (400 mesh) were investigated by a $120 \mathrm{kV}$ Philips CM12 microscope (FEI, Munich, Germany).

The gold-coated glass slide or prism was functionalized with the respective alkanethiol monolayer by self-assembly, using $200 \mu \mathrm{M}$ alkanethiol solution in ethanol. A structured self-assembled monolayer was obtained by microcontact printing with a PDMS-stamp of a squared structure.

Before measurement, the SPR prism or slide was cleaned with ethanol and dried under nitrogen flow. A stable SPR signal was obtained after flowing degassed water for about one hour. Each nanoparticle solution was cycled over the surface for six minutes. Next, degassed water was applied to the surface to remove the excess of nanoparticles from the slide. All steps were performed at a constant flow rate of $0.2 \mathrm{~mL} \mathrm{~min}^{-1}$. After the highest nanoparticle concentration was applied, the slide was extensively washed, at a flow rate of $0.4 \mathrm{~mL} \mathrm{~min}^{-1}$, until the signal intensity was back to its original level. At last, the SPR signal was calibrated to $\mathrm{RIU}$ with $\mathrm{NaCl}$ solutions of a known refractive index.

\section{Results}

In SPR imaging, the sensitivity is mostly influenced by the choice of a proper light source and by a camera system with a high resolution. The wavelength sensitivity of the SPR signal demands a nearly monochromatic, wavelength-stabilized light source. 
Typical monochromatic, polarized light sources are lasers. However, lasers do have spatial coherence, which causes speckles on rough surfaces and interference patterns from every dust particle and the edges of optical components in its light path [25]. Hence, an LEDbased light source was implemented to overcome these limitations. The optimized setup uses a 16-bit camera which yields a relative signal change of 1545 counts by measuring two aqueous reference solutions with a refractive index change of $1.2 \times 10^{-4} \mathrm{RIU}$, which equals a resolution of $7.8 \times 10^{-8} \mathrm{RIU}$ per count. When taking into account the signal's standard deviation ( $3 \sigma$ ) of 2.36 during the 3 min measurement time, a sensitivity of $5.5 \times 10^{-7}$ RIU can be achieved.

Such a low limit of detection enables SPRi as a sensing tool for the label-free detection of nanoparticles in water samples. To prove this, lanthanide-doped yttrium fluoride particles were studied in this work. The reason for choosing these particles is due to the possibility to synthesize them in a controlled size with high uniformity, as demonstrated by the low polydispersity, which allows one to calculate concentrations in a more comfortable way. These particles have a surface that is known to be highly attractive to negatively charged ligands, which saturate vacancies at the nanoparticle interface. As the surface ligand, $\mathrm{BF}_{4}{ }^{-}$was chosen, which increases their dispersibility in water. The nanoparticles have a size of $29 \pm 2 \mathrm{~nm}$, estimated by averaging the diameter of 3603 particles from transmission electron micrographs. This size was chosen as a perfect average size, as most nanoparticles in practical applications currently are in a range from 10 to $50 \mathrm{~nm}$ in diameter. The zeta-potential of these particles, measured at a concentration of $12.1 \pm 0.2 \mathrm{mg} \mathrm{mL}^{-1}$ in water, is $-3.63 \pm 0.09 \mathrm{mV}$, which is close to neutral, expecting that electrostatic interaction will not be the main force for binding to any surface.

To enable controllable interaction of the nanoparticles with the sensor surface, a suitable surface modification has to be found. Besides the surface coating, but according to the zeta-potential, their affinity was highest for the bare gold surface and a dodecanethiolmodified gold surface, with a neutral surface charge (Figure 1).

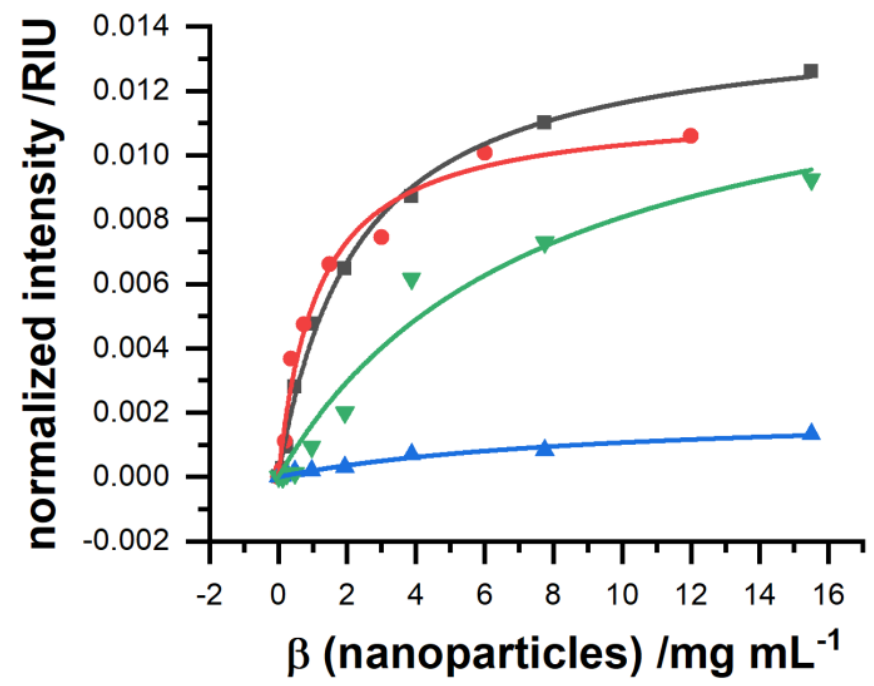

Figure 1. Dose-response curves of lanthanide-doped nanoparticles of $\mathrm{NaYF}_{4}$ type measured by a point-based SPR setup $(\mathrm{N}=1)$. The gold slides were functionalized by a self-assembled monolayer of dodecanethiol (red), 16-mercaptohexadecanoic acid (green) or aminohexanethiol (blue). The signal response to a non-modified, blank gold slide (black) is shown as a reference. The data have been fit by a Langmuir isotherm.

Thereby, the overall binding capacity was higher for blank gold, but the affinity was superior towards the self-assembled monolayer. The binding constants for all surface modifications are summarized in Table 1. In comparison, if surface charges are applied, negative via a coating of 16-mercaptohexadecanoic acid and positive via a coating of 6-aminohexanethiol, the binding performance was severely decreased. A negative sur- 
face charge decreases binding affinity but still enables a relatively high binding capacity, whereas a positive surface charge decreases both to a minimum.

Table 1. Binding constants of the different self-assembled monolayer-modified SPR surfaces and a blank gold surface to lanthanide-doped nanoparticles of $\mathrm{NaYF}_{4}$ type. A Langmuir isotherm was applied to the respective dose-response curves for fitting.

\begin{tabular}{cccc}
\hline Surface & $\mathbf{K}_{\mathbf{D}} \mathbf{( \mathbf { m g ~ } \mathbf { ~ m L } ^ { - \mathbf { 1 } } )}$ & $\left.\mathbf{K}_{\mathbf{a}} \mathbf{~} \mathbf{m g}^{-\mathbf{1}} \mathbf{~} \mathbf{L}\right)$ & $\mathbf{R}_{\mathbf{e q}}\left(\mathbf{1 0}^{-\mathbf{3}} \mathbf{R I U}\right)$ \\
\hline blank & $2.2 \pm 0.2$ & $0.44 \pm 0.04$ & $14.3 \pm 0.4$ \\
dodecanethiol & $1.1 \pm 0.2$ & $0.9 \pm 0.2$ & $11.5 \pm 0.7$ \\
16-mercaptohexadecanoic acid & $7.7 \pm 3$ & $0.13 \pm 0.05$ & $14 \pm 3$ \\
aminohexanethiol & $9.6 \pm 3$ & $0.10 \pm 0.03$ & $2.1 \pm 0.3$ \\
\hline
\end{tabular}

The order of the different binding affinities cannot be explained by electrostatic interactions between the sensor surface and the nanoparticle only. Here, one would expect that amino-functionalized surface would lead to good binding. Indeed, binding can be found, but the other surfaces are superior. For surfaces with carboxy functionality at the surface, the particles show a better adsorption behavior as their surface consists of lanthanide ions which do form a stable coordinative binding to carboxy groups, which has been reviewed by Wolfbeis et al. [30]. Surprisingly, dodecanethiol was also able to bind the nanoparticles. This finding is interesting as it suggests that the surface modification of those particles performed by ligand exchange, where oleate molecules at the particle surface get stripped off, does result in a complete loss of the original surface capping. The reason for the binding might be that some oleate is still present at the particle surface which interacts by the intercalation of the long hydrophobic tail of the oleate with the long hydrophobic chain of the dodecanethiol. Better insights into nanoparticle surface interaction are currently under investigation and are out of scope of this work. It seems reasonable to continue with the dodecanethiol coating in further measurements. Figure 2 shows a typical measurement sequence. After each binding step, a short washing step was applied to exclude the unbound particles from the SPR signal. The particles show a high overall signal change, which is due to their high mass and size.

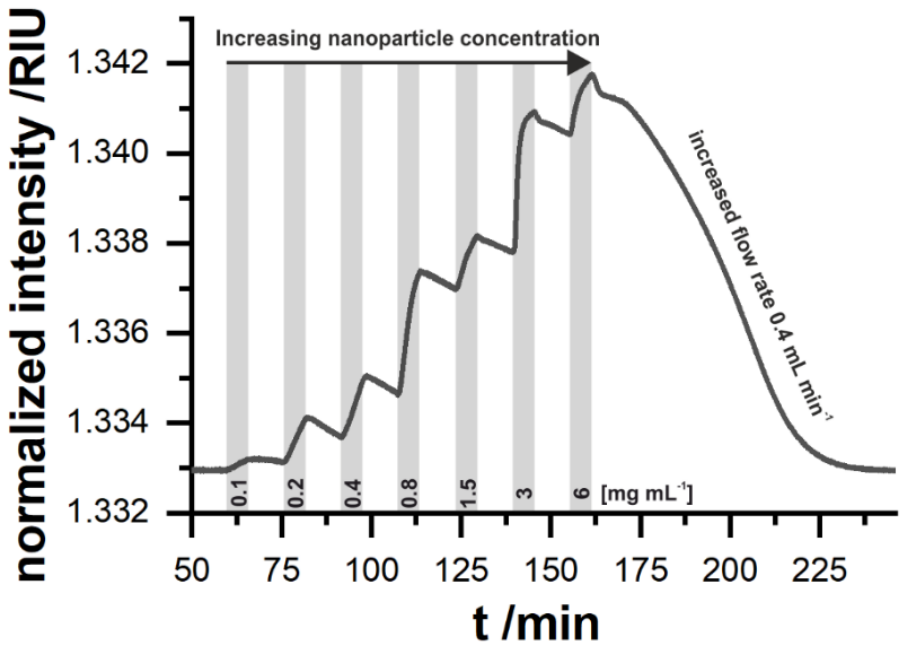

Figure 2. Time course of a SPR measurement sequence. The grey areas indicate the presence of lanthanide-doped nanoparticles of $\mathrm{NaYF}_{4}$ type in the measurement solution, whereas in the white areas, the system was rinsed with water. In the first part, until $t=160 \mathrm{~min}$, a flow rate of $0.2 \mathrm{~mL} \mathrm{~min}^{-1}$ was applied, which was then increased to $0.4 \mathrm{~mL} \mathrm{~min}^{-1}$. The gold surface was modified by a dodecanethiol monolayer.

For an online sensing system of environmental samples such as freshwater, reversibility is one of the key requirements. Gold slides modified with a dodecanethiol monolayer 
can achieve this. SPR measurements at a flow rate of $0.2 \mathrm{~mL} \mathrm{~min}^{-1}$ allow us to easily discriminate the presence of $0.1 \mathrm{mg} \mathrm{mL}^{-1}$ nanoparticles in water. In contrast to changes in the bulk refractive index, the slope in the change in the signal is concentration-dependent, which also indicates that the particles adsorb to the surface by means of a weak interaction. The desorption at the same flow rate is somehow slower compared to the absorption of the particles to the surface. When doubling the flow rate, the sensor surface can be regenerated within about one hour. This would be attractive for stop flow sensing systems, which would be able to get one data point in less than $1.5 \mathrm{~h}$. Such a behavior is beneficial for a long-term continuous sensor, since a high signal response can be obtained and the adsorption is faster than the desorption which makes a regeneration step expendable. An optimization of the microfluidic flow cell together with the flow rate is expected to improve the sample rate, but this is out of scope of this study. The discrimination of the signal change caused by fluctuations in the refractive index of the media itself and the presence of nanoparticles can be achieved by exploiting the binding behavior of different surface modifications together with pattern analysis. This requires the use of sensor arrays and SPR imaging.

SPR imaging relying on an LED and a camera shows the characteristic of an angle shift over the image in $x$-direction, which was observable during this measurement (Figure 3). This behavior would exclude certain regions to be used for signal generation if the whole imaging surface area should be used for only one receptor. However, this would contravene the potential of SPRi. If multiple receptors are used in SPRi, this circumstance can be even useful. Each recognition element has a different SPR angle, as seen for dodecanethiol and aminohexanethiol in Figure 3c. The angle shift can be exploited to place both receptors in a region where the complete linear intensity range from the minimum to total internal reflection can be used.

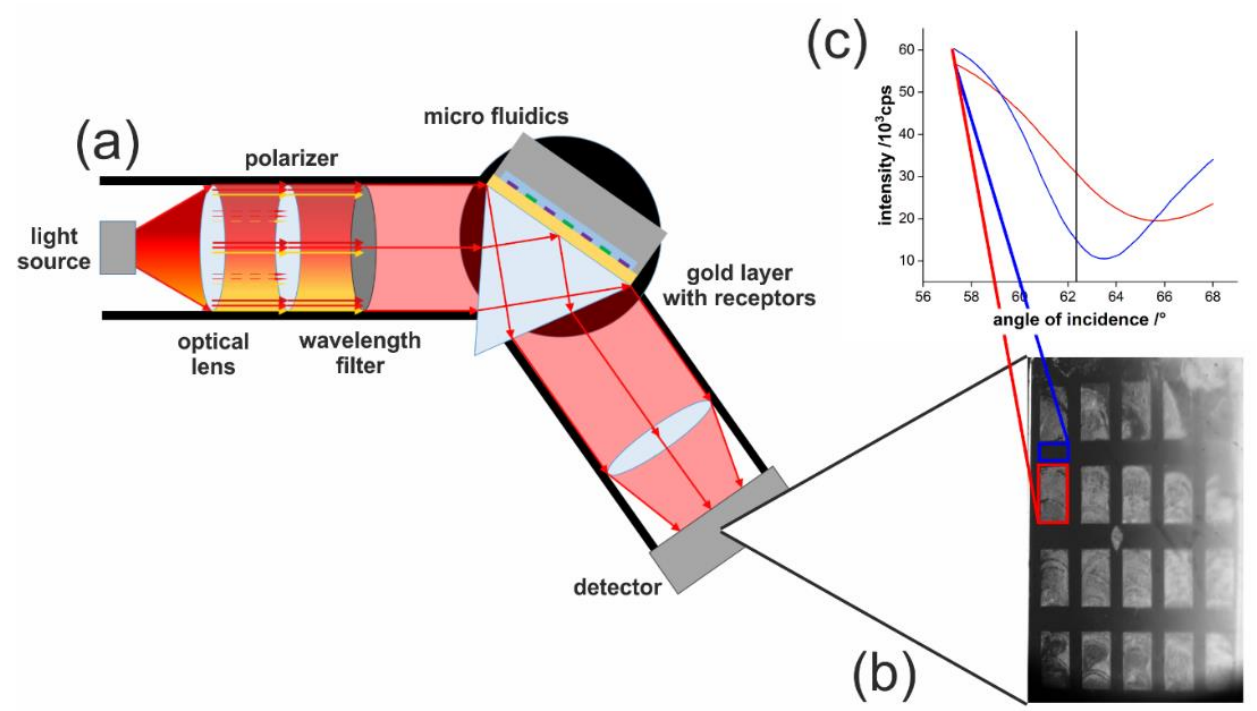

Figure 3. (a) Scheme of the SPRi setup. (b) SPR image obtained when coated with dodecanethiol squares (blue) and surrounded by aminohexanethiole (red). The irregular coating is caused by the stamping procedure to form a patterned surface. (c) SPR curves from the two respective thiols. Both were obtained within the region of interest with the same dimensions at the same $x$-position. The marked positions only show the principal division for illustration purposes and do not equal the actual positions.

SPR imaging is needed to further minimize the detection limit as well. The association curves measured by a commercially available SPR device, shown in Figure 4, feature a limit of detection of $29 \pm 9 \mu \mathrm{g} \mathrm{mL}-1$, which still is too high for a practical application. In contrast to this, the optimized homebuilt SPRi system with an LED light source was able to improve the limit of detection under identical measurement conditions by a factor 
of 20 (Figure 4). It should be said that, due to measurement, there was a reduction in the number of nanoparticles of around 50\% after multiple measurement cycles, due to experimental limitations, which were both identical for both measurements systems. For a fair comparison, the limit of detection was calculated based on the ICP-OES-estimated concentration of the nanoparticle applied to each system without prior application.

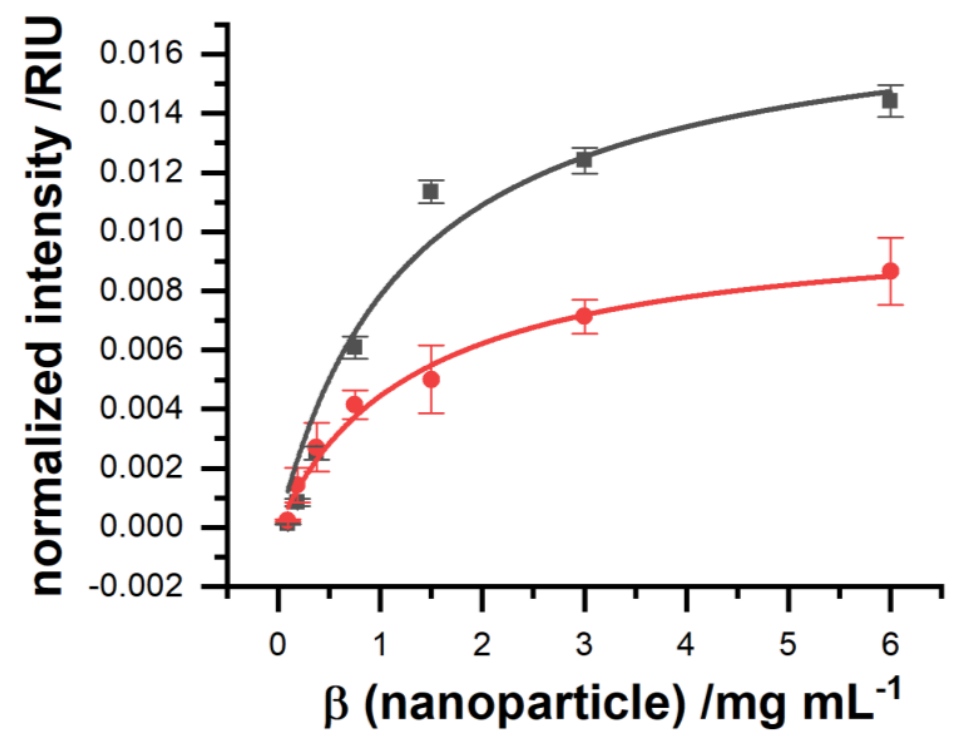

Figure 4. Dose-response curve of $\mathrm{NaYF}_{4}$-based nanoparticles measured by a point-based SPR setup (red) and an SPR imaging setup with LED illumination (black) $(\mathrm{N}=4)$.

Detailed analysis found that the equilibrium dissociation constant stayed the same, but aside from that, the equilibrium binding capacity was increased (Table 2), leading to a limit of detection (LOD) of $1.5 \mu \mathrm{g} \mathrm{mL}^{-1}$. In contrast to a point-based SPR, where the signal is averaged from the whole illuminated area on the gold, the imaging setup allows us to select only those pixels on the camera image which are best suited in terms of sensitivity and noise due to irregularities and artifacts such as small air bubbles, which get stuck over time when analyzing real samples.

Table 2. LODs and binding constants of the point-based SPR and the optimized SPRi setup of $\mathrm{NaYF}_{4}$ nanoparticle on a dodecanethiol-coated surface. A Langmuir isotherm was applied to the respective dose-response curves for fitting.

\begin{tabular}{cccc}
\hline Setup & LOD $\left(\mu \mathbf{g ~ m L} \mathbf{~}^{-\mathbf{1}}\right)$ & $\mathbf{K}_{\mathbf{D}}\left(\mathbf{m g ~} \mathbf{~ L L}^{-\mathbf{1}}\right)$ & $\mathbf{R}_{\mathbf{e q}}\left(\mathbf{1 0}^{-\mathbf{3}} \mathbf{R I U}\right)$ \\
\hline Point-based SPR & $29 \pm 9$ & $1.3 \pm 0.2^{*}$ & $10.4 \pm 0.6^{*}$ \\
SPR imaging & $1.5 \pm 0.7$ & $1.3 \pm 0.4$ & $17.9 \pm 0.6$ \\
\hline *Value differs from that in Table 1, due to another batch of similar produced nanoparticles was used.
\end{tabular}

\section{Discussion}

In this work, we demonstrated that the major drawback of reflectivity-based SPRi-its insufficient sensitivity — can be overcome by improving the camera system for detection, as well as the light source. For further development, such a system is capable for miniaturization, and therefore for a wide range of sensing applications. The spreading of the angle of incidence in one direction of the image collected by LED-based SPRi is not necessarily a drawback, as it enables the simultaneous use of different angles of incidence (in a range of up to $3^{\circ}$ in our setup) within one sensor chip, without the utilization of any moving component. This allows us to place every receptor with its individual refractive index to be placed at its most sensitive angle position. Temperature drift and fluctuations in the light source can be overcome by using internal reference spots placed on the gold slide. This work is currently under investigation. 
By the modification of the sensor surface with different self-assembled monolayers, it became apparent that the interaction of nanoparticles with a sensor surface is of a complex nature, as nanoparticles will be coated by additional surface ligands. Therefore, sensor arrays with cross-reactivity receptors are suggested to tackle this challenge by means of pattern recognition. Surface plasmon resonance imaging might be capable of handling a mix of different particles in composition and size, since it can measure multiple analytes simultaneously. This is made possible by the combination of different receptors and by confining the plasmonic field in its penetration depth by applying nanostructured gold surfaces [31,32]. Thereby, the key will not be to find the most selective receptor for each particle, but to find a smart combination of low selective receptors, which can detect particles by their specific signal pattern. Using LED-based SPRi for this approach, each receptor can be positioned at the angle of highest sensitivity, due to the tunable angle shift. The limit of detection still needs to be improved when applications in the quality control of environmental samples are envisioned.

Author Contributions: Conceptualization, L.W., T.H., P.H. and R.B.; methodology, P.H., L.W., S.M.; validation, L.W., P.H., S.M.; investigation, L.W., P.H., S.M.; data curation, L.W. and P.H.; writingoriginal draft preparation, L.W., P.H.; writing-review and editing, L.W., P.H. and T.H.; supervision, R.B. and T.H.; All authors have read and agreed to the published version of the manuscript.

Funding: This work is funded by Bavarian Ministry of Economic Affairs, Energy and Technology (ESB051/006).

Institutional Review Board Statement: Not applicable.

Informed Consent Statement: Not applicable.

Data Availability Statement: Not applicable.

Conflicts of Interest: The authors declare no conflict of interest. The funders had no role in the design of the study; in the collection, analyses, or interpretation of data; in the writing of the manuscript, or in the decision to publish the results.

\section{References}

1. EC. Types and Uses of Nanomaterials, Including Safety Aspects; European Commission: Brussels, Belgium, 2012.

2. Giese, B.; Klaessig, F.; Park, B.; Kaegi, R.; Steinfeldt, M.; Wigger, H.; von Gleich, A.; Gottschalk, F. Risks, Release and Concentrations of Engineered Nanomaterial in the Environment. Sci. Rep. 2018, 8, 1565. [CrossRef]

3. Botta, C.; Labille, J.; Auffan, M.; Borschneck, D.; Miche, H.; Cabié, M.; Masion, A.; Rose, J.; Bottero, J.Y. TiO 2 -based nanoparticles released in water from commercialized sunscreens in a life-cycle perspective: Structures and quantities. Environ. Pollut. 2011, 159, 1543-1550. [CrossRef]

4. Blinova, I.; Ivask, A.; Heinlaan, M.; Mortimer, M.; Kahru, A. Ecotoxicity of nanoparticles of $\mathrm{CuO}$ and $\mathrm{ZnO}$ in natural water. Environ. Pollut. 2010, 158, 41-47. [CrossRef]

5. Gao, Y.; Yang, T.; Jin, J. Nanoparticle pollution and associated increasing potential risks on environment and human health: A case study of China. Environ. Sci. Pollut. Res. 2015, 22, 19297-19306. [CrossRef] [PubMed]

6. Troester, M.; Brauch, H.-J.; Hofmann, T. Vulnerability of drinking water supplies to engineered nanoparticles. Water Res. 2016, 96, 255-279. [CrossRef] [PubMed]

7. Zhang, M.; Yang, J.; Cai, Z.; Feng, Y.; Wang, Y.; Zhang, D.; Pan, X. Detection of engineered nanoparticles in aquatic environments: Current status and challenges in enrichment, separation, and analysis. Environ. Sci. Nano 2019, 6, 709-735. [CrossRef]

8. Pollard, M.R.; Sparnacci, K.; Wacker, L.J.; Kerdoncuff, H. Polymer nanoparticle identification and concentration measurement using fiber-enhanced raman spectroscopy. Chemosensors 2020, 8, 21. [CrossRef]

9. Neves, M.M.; Nouws, H.P.; Delerue-Matos, C.; Martín-Yerga, D. Electrochemical detection and characterization of nanoparticles: A potential tool for environmental purposes. Curr. Opin. Electrochem. 2020, 22, 58-64. [CrossRef]

10. Homola, J.; Yee, S.S.; Gauglitz, G. Surface plasmon resonance sensors. Sens. Actuator B Chem. 1999, 54, 3-15. [CrossRef]

11. Nguyen, H.H.; Park, J.; Kang, S.; Kim, M. Surface plasmon resonance: A versatile technique for biosensor applications. Sensors 2015, 15, 10481-10510. [CrossRef]

12. Amirjani, A.; Rahbarimehr, E. Recent advances in functionalization of plasmonic nanostructures for optical sensing. Microchim. Acta 2021, 188, 1-17. [CrossRef] [PubMed]

13. Masson, J.F. Portable and field-deployed surface plasmon resonance and plasmonic sensors. Analyst 2020, 145, 3776-3800. [CrossRef] [PubMed] 
14. Nizamov, S.; Scherbahn, V.; Mirsky, V.M. Detection and quantification of single engineered nanoparticles in complex samples using template matching in wide-field surface plasmon microscopy. Anal. Chem. 2016, 88, 10206-10214. [CrossRef] [PubMed]

15. Scherbahn, V.; Nizamov, S.; Mirsky, V.M. Plasmonic detection and visualization of directed adsorption of charged single nanoparticles to patterned surfaces. Microchim. Acta 2016, 183, 2837-2845. [CrossRef]

16. Jiang, D.; Chen, H.B.; Qian, C.; Zhou, X.L.; Liu, X.W. Determining the Aggregation Kinetics of Nanoparticles by Single Nanoparticle Counting. ACS EST Water 2021, 1, 672-679. [CrossRef]

17. Kuzmichev, A.; Skolnik, J.; Zybin, A.; Hergenröder, R. Absolute analysis of nanoparticle suspension with surface plasmon microscopy. Anal. Chem. 2018, 90, 10732-10737. [CrossRef]

18. Grześkowiak, B.F.; Tuśnio, K.; Woźniak, A.; Szalata, M.; Lipiński, D.; Jurga, S.; Słomski, R. Transgenic Plant Detection Using an AuNPs Based SPR Biosensor. Biosensors 2019, 9, 116. [CrossRef]

19. Fenzl, C.; Hirsch, T.; Baeumner, A.J. Liposomes with high refractive index encapsulants as tunable signal amplification tools in surface plasmon resonance spectroscopy. Anal. Chem. 2015, 87, 11157-11163. [CrossRef]

20. Fournel, A.; Mantel, M.; Pinger, M.; Manesse, C.; Dubreuil, R.; Herrier, C.; Rousselle, T.; Livache, T.; Bensafi, M. An experimental investigation comparing a surface plasmon resonance imaging-based artificial nose with natural olfaction. Sens. Actuator B Chem. 2020, 320, 128342. [CrossRef]

21. Maho, P.; Herrier, C.; Livache, T.; Rolland, G.; Comon, P.; Barthelme, S. Reliable chiral recognition with an optoelectronic nose. Biosens. Bioelectron. 2020, 159, 112183. [CrossRef]

22. Wang, D.; Loo, J.F.C.; Chen, J.; Yam, Y.; Chen, S.-C.; He, H.; Kong, S.K.; Ho, H.P. Recent Advances in Surface Plasmon Resonance Imaging Sensors. Sensors 2019, 19, 1266. [CrossRef]

23. Wong, C.L.; Olivo, M. Surface Plasmon Resonance Imaging Sensors: A Review. Plasmonics 2014, 9, 809-824. [CrossRef]

24. Hausler, P.; Roth, C.; Vitzthumecker, T.; Bierl, R. Miniaturized Surface Plasmon Resonance Based Sensor Systems-Opportunities and Challenges. In Optics, Photonics and Laser Technology 2018. Springer Series in Optical Sciences; Ribeiro, P., Raposo, M., Eds.; Springer: Cham, Switzerland, 2019; Volume 223, pp. 169-195.

25. Hausler, P.; Jobst, S.; Fischer, J.; Roth, C.; Bierl, R. Homogeneous Light Source for Surface Plasmon Resonance Imaging. In Proceedings of the 8th International Conference on Photonics, Optics and Laser Technology (PHOTOPTICS 2020), 1st ed.; Albella, P., Raposo, M., Andrews, D., Ribeiro, P., Eds.; Scitepress: Sebútal, Portugal, 2021; pp. 163-167.

26. Sokolova, V.I.; Zvyagina, A.V.; Igumnovb, S.M.; Molchanovaa, S.I.; Nazarova, M.M.; Nechaevc, A.V.; Savelyeva, A.G.; Tyutyunovb, A.A.; Khaydukova, E.V.; Panchenko, V.Y. Determination of the Refractive Index of $\beta-\mathrm{NaYF}_{4} / \mathrm{Yb}^{3+} / \mathrm{Er}^{3+} / \mathrm{Tm}^{3+} \mathrm{Nanocrystals}$ Using Spectroscopic Refractometry. Opt. Spectrosc. 2014, 118, 609-613. [CrossRef]

27. Khlebtsov, B.N.; Khanadeev, V.A.; Khlebtsov, N.G. Determination of the Size, Concentration, and Refractive Index of Silica Nanoparticles from Turbidity Spectra. Langmuir 2008, 24, 8964-8970. [CrossRef]

28. Märkl, S.; Schroter, A.; Hirsch, T. Small and Bright Water-Protected Upconversion Nanoparticles with Long-Time Stability in Complex, Aqueous Media by Phospholipid Membrane Coating. Nano Lett. 2020, 20, 8620-8625. [CrossRef] [PubMed]

29. Himmelstoß, S.F.; Hirsch, T. Long-term Colloidal and Chemical Stability in Aqueous Media of $\mathrm{NaYF}_{4}{ }^{-} \mathrm{Type}_{\mathrm{U}} \mathrm{Upconversion}^{-}$ Nanoparticles Modified by Ligand-Exchange. Part. Part. Syst. Charact. 2019, 36, 1900235. [CrossRef]

30. Muhr, V.; Wilhelm, S.; Hirsch, T.; Wolfbeis, O.S. Upconversion nanoparticles: From hydrophobic to hydrophilic surfaces. Acc. Chem. Res. 2014, 47, 3481-3493. [CrossRef] [PubMed]

31. Genslein, C.; Hausler, P.; Kirchner, E.M.; Bierl, R.; Baeumner, A.J.; Hirsch, T. Graphene-enhanced plasmonic nanohole arrays for environmental sensing in aqueous samples. Beilstein J. Nanotechnol. 2016, 7, 1564-1573. [CrossRef]

32. Couture, M.; Live, L.S.; Dhawan, A.; Masson, J.F. EOT or Kretschmann configuration? Comparative study of the plasmonic modes in gold nanohole arrays. Analyst 2012, 137, 4162-4170. [CrossRef] [PubMed] 